\title{
Cardiovascular Risk and Events in 17 Low-, Middle-, and High-Income Countries
}

\author{
S. Yusuf, S. Rangarajan, K. Teo, S. Islam, W. Li, L. Liu, J. Bo, Q. Lou, F. Lu, T. Liu, \\ L. Yu, S. Zhang, P. Mony, S. Swaminathan, V. Mohan, R. Gupta, R. Kumar, \\ K. Vijayakumar, S. Lear, S. Anand, A. Wielgosz, R. Diaz, A. Avezum, \\ P. Lopez-Jaramillo, F. Lanas, K. Yusoff, N. Ismail, R. Iqbal, O. Rahman, \\ A. Rosengren, A. Yusufali, R. Kelishadi, A. Kruger, T. Puoane, A. Szuba, \\ J. Chifamba, A. Oguz, M. McQueen, M. McKee, and G. Dagenais, \\ for the PURE Investigators*
}

The authors' full names, academic degrees, and affiliations are listed in the Appendix. Address reprint requests to Dr. Yusuf at the Population Health Research Institute, DBCVSRI, Hamilton General Hospital, 237 Barton St. East, Hamilton, ON L8L 2X2, Canada, or at yusufs@mcmaster.ca.

*A complete list of the investigators in the Prospective Urban Rural Epidemiologic (PURE) study is provided in the Supplementary Appendix, available at NEJM.org.

N EnglJ Med 2014;371:818-27. DOI: 10.1056/NEJMoal311890

Copyright () 2014 Massachusetts Medical Society.

\section{A BSTRACT}

\section{BACKGROUND}

More than $80 \%$ of deaths from cardiovascular disease are estimated to occur in low-income and middle-income countries, but the reasons are unknown.

\section{METHODS}

We enrolled 156,424 persons from 628 urban and rural communities in 17 countries (3 high-income, 10 middle-income, and 4 low-income countries) and assessed their cardiovascular risk using the INTERHEART Risk Score, a validated score for quantifying risk-factor burden without the use of laboratory testing (with higher scores indicating greater risk-factor burden). Participants were followed for incident cardiovascular disease and death for a mean of 4.1 years.

\section{RESULTS}

The mean INTERHEART Risk Score was highest in high-income countries, intermediate in middle-income countries, and lowest in low-income countries $(\mathrm{P}<0.001)$. However, the rates of major cardiovascular events (death from cardiovascular causes, myocardial infarction, stroke, or heart failure) were lower in high-income countries than in middle- and low-income countries (3.99 events per 1000 personyears vs. 5.38 and 6.43 events per 1000 person-years, respectively; $\mathrm{P}<0.001)$. Case fatality rates were also lowest in high-income countries $(6.5 \%, 15.9 \%$, and $17.3 \%$ in high-, middle-, and low-income countries, respectively; $\mathrm{P}=0.01$ ). Urban communities had a higher risk-factor burden than rural communities but lower rates of cardiovascular events ( 4.83 vs. 6.25 events per 1000 person-years, $\mathrm{P}<0.001$ ) and case fatality rates $(13.52 \%$ vs. $17.25 \%, \mathrm{P}<0.001)$. The use of preventive medications and revascularization procedures was significantly more common in high-income countries than in middle- or low-income countries $(\mathrm{P}<0.001)$.

\section{CONCLUSIONS}

Although the risk-factor burden was lowest in low-income countries, the rates of major cardiovascular disease and death were substantially higher in low-income countries than in high-income countries. The high burden of risk factors in highincome countries may have been mitigated by better control of risk factors and more frequent use of proven pharmacologic therapies and revascularization. (Funded by the Population Health Research Institute and others.) 
W

ORLDWIDE, 18 MILLION DEATHS ANnually are attributed to cardiovascular diseases. ${ }^{1}$ From the 1930 s to the 1950 s, the rate of cardiovascular disease increased in high-income countries, but during this period, the rates were low in middle- and low-income countries. $^{2,3}$ Since the mid-1970s, the rate of death from cardiovascular diseases has declined markedly in several high-income countries, owing to reductions in risk factors and improved management of cardiovascular disease. ${ }^{4}$ By contrast, the incidence of cardiovascular disease has been increasing in some low-income and middle-income countries, ${ }^{5,6}$ with $80 \%$ of the global burden estimated to occur in these countries. ${ }^{1}$ It is not known whether the risk-factor burden and the incidence of cardiovascular disease are now higher in lowand middle-income countries than in high-income countries, whether mortality after a cardiovascular event is higher in low- and middle-income countries than in high-income countries, or whether both are true.

To address these questions, we initiated the Prospective Urban Rural Epidemiologic (PURE) cohort study involving more than 150,000 adults in 17 high-, middle-, and low-income countries. We now report the risk-factor burden, the incidence of cardiovascular disease, related case fatality rates, and mortality over the course of 4.1 years of follow-up.

METHODS

\section{STUDY DESIGN}

Details of the design and methods of the PURE study have been published previously ${ }^{7-10}$ and are summarized in the Supplementary Appendix, available with the full text of this article at NEJM .org. Briefly, we prospectively recruited 156,424 persons from 628 communities in 17 low-, middle-, and high-income countries (Table S1 in the Supplementary Appendix). ${ }^{7-10}$ Recruitment began in January 2003. We selected the number and locations of countries with a view toward balancing the goal of including a large number of communities in countries with a wide range of socioeconomic circumstances with the goal of recruiting participants for whom successful longterm follow-up would be feasible. It was not considered practical to implement strict proportionate sampling of the whole world, any specific country, or any specific region.
Using World Bank classifications of the income levels of countries at the time the PURE study was started, we included 4 low-income countries (Bangladesh, India, Pakistan, and Zimbabwe), 10 middle-income countries (Argentina, Brazil, Chile, China, Colombia, Iran, Malaysia, Poland, South Africa, and Turkey), and 3 high-income countries (Canada, Sweden, and the United Arab Emirates). In each country, we selected urban and rural communities within participating sites on the basis of prespecified guidelines ${ }^{7-10}$ (Table S2 in the Supplementary Appendix).

Households within a community were selected with the goal of achieving a broadly representative sample of that community (see the Supplementary Appendix for details). A household was eligible if at least one member was 35 to 70 years of age and the members of the household intended to remain at the current address for at least 4 more years. All eligible persons who provided written informed consent were enrolled (response rate, $78 \%$ ).

By including persons from both urban and rural communities in low-, middle-, and highincome countries on five continents, we expected substantial variations in health determinants and outcomes. Information concerning the representativeness of the PURE cohort is provided in Figures S1 through S4 and the Methods section in the Supplementary Appendix.

\section{STUDY OVERSIGHT AND MANAGEMENT}

The study was designed by the first author and supervised by the first two authors together with each country's designated national leader (see the Supplementary Appendix). The PURE study was funded by nonprofit, governmental, and industry sponsors. The funders of the study had no role in the design or conduct of the study, in the collection, analysis, or interpretation of the data, or in the writing of the manuscript. The study was approved by the ethics committee at each participating center and at Hamilton Health Sciences, Hamilton, Ontario, Canada. All the participants provided written informed consent.

\section{DATA COLLECTION}

We collected data at national, community, household, and individual levels using standardized questionnaires. ${ }^{7}$ Questions about age, sex, education, smoking status, hypertension, diabetes, and obesity were identical to those in the INTERHEART 
and INTERSTROKE studies. ${ }^{11,12}$ Participants were considered to have hypertension if they had blood pressure higher than $140 / 90 \mathrm{~mm} \mathrm{Hg}$ or if they reported a history of hypertension. Participants were considered to have diabetes if they reported having diabetes or if they had a fasting glucose level higher than $7.0 \mathrm{mmol}$ per liter (126 mg per deciliter). A total cholesterol level higher than $5.2 \mathrm{mmol}$ per liter (201 $\mathrm{mg}$ per deciliter) was considered to be an elevated level.

Follow-up occurred between 2008 and 2013. Information on specific events was obtained from participants or their family members, and events were adjudicated centrally in each country by trained physicians who reviewed verbal (i.e., oral) autopsy reports ${ }^{13,14}$ and medical records with the use of standardized definitions. Major cardiovascular events included death from cardiovascular causes and nonfatal stroke, myocardial infarction, and heart failure. Nonmajor cardiovascular events included all other cardiovascular disease events that led to hospitalization. Details regarding the determination and adjudication of risk factors and end points are provided in the Supplementary Appendix.

\section{INTERHEART RISK SCORE}

To quantify the risk-factor burden, we calculated the INTERHEART Risk Score ${ }^{15}$ for each participant, using the version that did not include data on cholesterol levels. The INTERHEART Risk Score is a validated score that includes data on age; sex; status with respect to smoking, diabetes, high blood pressure, and family history of heart disease; waist-to-hip ratio; psychosocial factors; diet; and physical activity. Scores range from 0 to 48 , with higher scores indicating a greater risk-factor burden. Details concerning the development and validation of the INTERHEART Risk Score have been published previously ${ }^{15}$ and are also provided in the Supplementary Appendix; the scoring system itself is shown in Table S3 in the Supplementary Appendix, with validation information in Table S4 in the Supplementary Appendix.

\section{STATISTICAL ANALYSIS}

Means and confidence intervals were calculated according to urban and rural location and the economic status of the country. For all cardiovascular events and deaths, the time to an event was calculated to obtain incidence rates per 1000 person-years that were age-standardized after adjustment for sex. Case fatality rates were calculated for myocardial infarction, stroke, and heart failure after adjustment for duration of follow-up, age, and sex. A Cox proportional-hazards model was used to test for trends in the incidence of cardiovascular disease across high-income, middle-income, and low-income countries and between urban versus rural locations. Given the multiplicity of comparisons, $\mathrm{P}$ values should be interpreted cautiously, except when they are very small (e.g., $\mathrm{P}<0.001)$.

\section{RESULTS}

\section{STUDY PARTICIPANTS}

We identified 388,796 persons from 108,927 households in 628 communities (348 urban and 280 rural) in 17 countries on five continents. Recruitment started in India in 2003; however, most persons were recruited between 2005 and 2009. A total of 200,905 of the potential participants we identified (51.7\%) were eligible for the study, and 156,424 of the eligible persons (77.9\%) participated. Characteristics of eligible and enrolled persons were generally similar except for a slightly higher proportion of women and of persons with hypertension and diabetes in the latter group (Table S5 in the Supplementary Appendix). Table 1 shows the baseline characteristics of the enrolled participants.

\section{RISK-FACTOR BURDEN}

The mean INTERHEART Risk Score was highest in high-income countries $(12.89$; $95 \%$ confidence interval [CI], 12.79 to 12.98), intermediate in middle-income countries (10.47; 95\% CI, 10.43 to 10.50), and lowest in low-income countries (8.28; $95 \%$ CI, 8.23 to 8.34$)(\mathrm{P}<0.001)$ (Fig. 1). The INTERHEART Risk Score was higher in rural areas than in urban areas in high-income countries $(13.43$ vs. $12.67, \mathrm{P}<0.001)$ but lower in rural areas than in urban areas in middle-income countries $(10.11$ vs. $10.81, \mathrm{P}<0.001)$ and in lowincome countries (7.57 vs. 9.09, $\mathrm{P}<0.001$ ). Total cholesterol levels (Table 1) were highest in highincome countries, intermediate in middle-income countries, and lowest in low-income countries, but information on cholesterol levels was available for only about $75 \%$ of the participants.

\section{MEDICATIONS AND REVASCULARIZATION}

Among participants without cardiovascular disease at baseline, the use of antiplatelet drugs was highest in high-income countries, interme- 


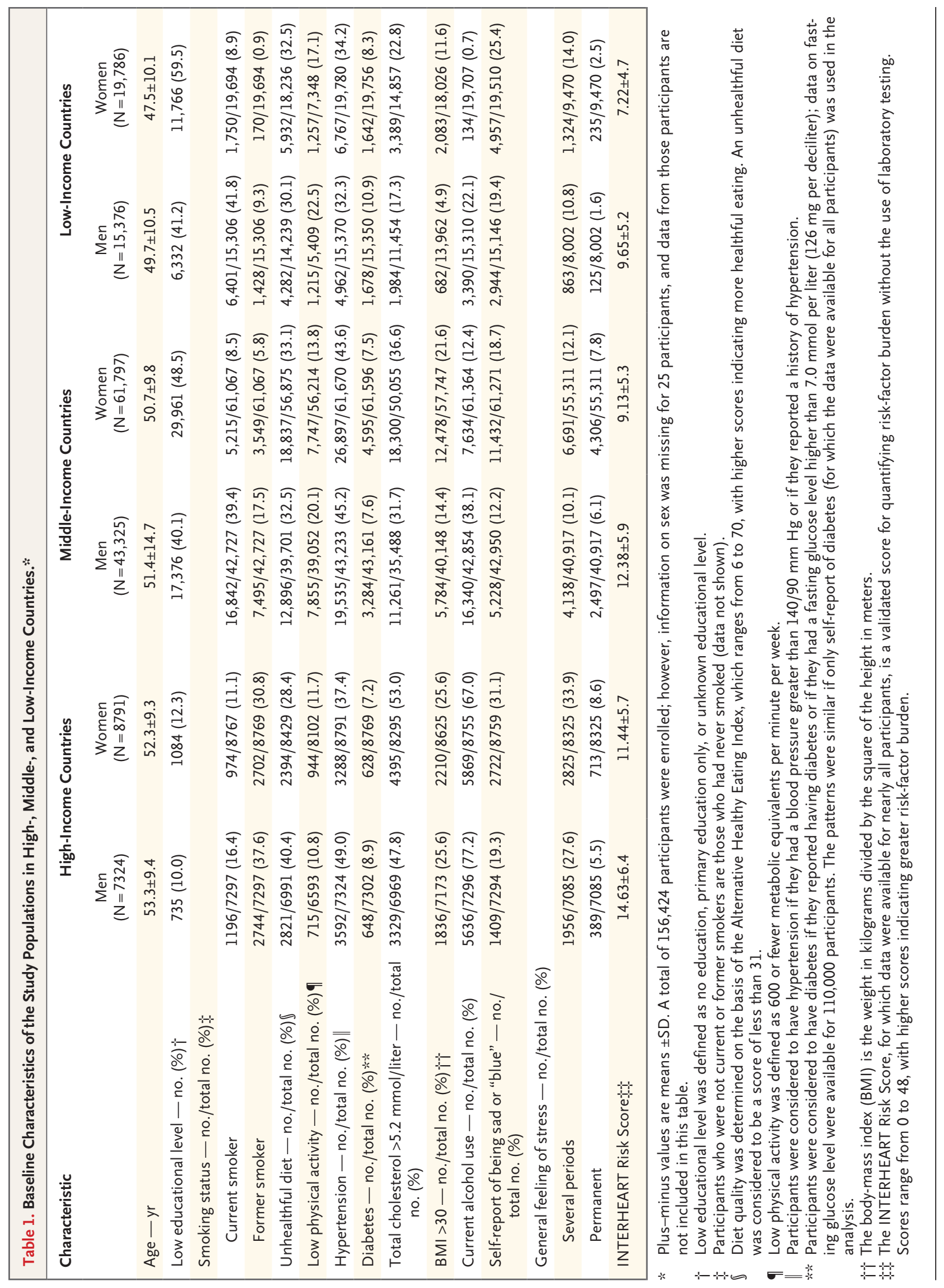

N ENGLJ MED 371;9 NEJM.ORG AUGUST 28, 2014 
diate in middle-income countries and lowest in low-income countries $(8.1 \%, 2.8 \%$, and $0.5 \%$, respectively); a similar pattern was seen in the use of beta-blockers $(4.5 \%, 3.0 \%$, and $1.8 \%$, respectively), blockers of the renin-angiotensin system (10.5\%, $4.8 \%$, and $0.8 \%$, respectively), and statins (10.3\%, $1.6 \%$, and $0.3 \%$, respectively). The patterns were similar among persons with prevalent cardiovascular disease. ${ }^{14}$ The frequency of coronary revascularization (either coronary-artery bypass grafting or percutaneous coronary intervention) was highest in high-income countries, intermediate in middle-income countries, and lowest in low-income countries.

\section{FOLLOW-UP}

The mean duration of follow-up was 4.1 years. The rate of complete follow-up was $92.8 \%$ (97.5\% in high-income countries, $94.4 \%$ in middle-income countries, and $85.9 \%$ in low-income countries). An additional $4.8 \%$ of the participants had partial follow-up information, and $2.4 \%$ had no followup information available. Follow-up rates were similar in urban and rural communities in highincome countries $(97.2 \%$ and $98.1 \%$, respectively) and middle-income countries $(94.2 \%$ and $94.5 \%$, respectively) but were lower in urban communities than in rural communities in low-income countries ( $76.8 \%$ vs. $94.0 \%)$. Characteristics of all the study participants, as compared with participants with follow-up information, are shown in Table S6 in the Supplementary Appendix.

\section{OVERALL EVENTS}

There were 3900 deaths among 152,463 participants with confirmed vital status (2.6\%). Of the 152,606 participants with complete or partial follow-up data, 1736 had a myocardial infarction (1.1\%), 1317 had a stroke (0.9\%), 414 had heart failure $(0.3 \%)$, and 3483 had at least one major cardiovascular event (2.3\%). An additional 1163 participants $(0.8 \%)$ were hospitalized for other cardiovascular reasons (termed nonmajor cardiovascular events). In total, 4646 participants (3.0\%) had at least one cardiovascular event.

CARDIOVASCULAR EVENTS ACCORDING TO COUNTRY INCOME STRATUM

There were 216 deaths (2.43 per 1000 personyears of follow-up) in high-income countries, 2052 deaths (5.59 per 1000 person-years of follow-up) in middle-income countries, and 1632 deaths $(9.23$ per 1000 person-years of follow-up) in low-income

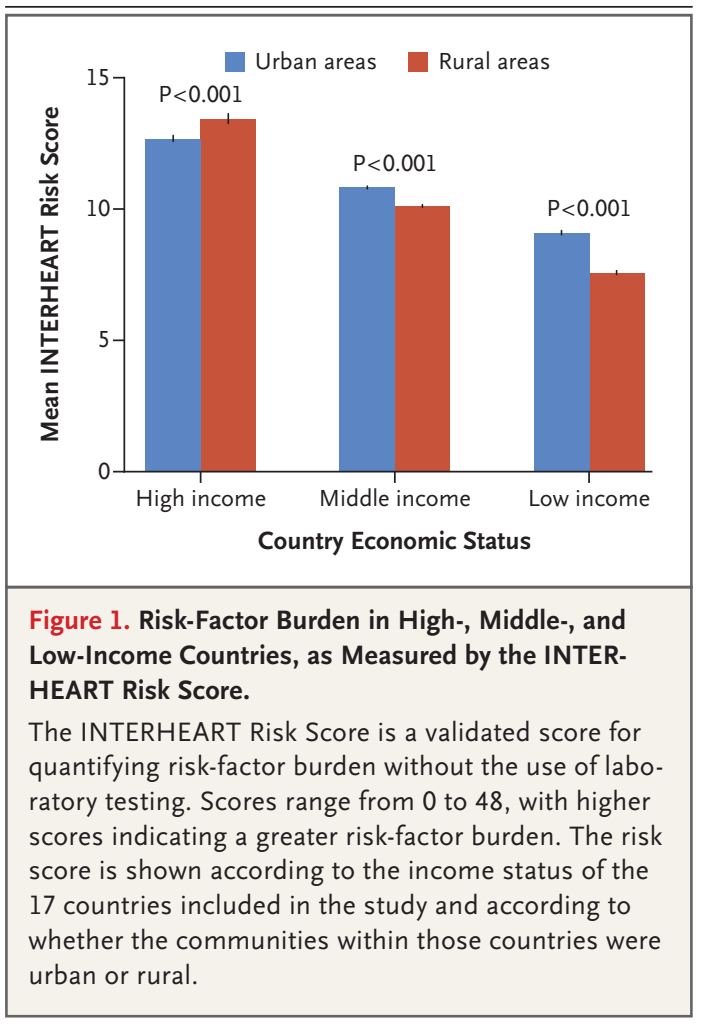

countries ( $\mathrm{P}<0.001$ for trend) (Fig. 2, and Table S7 in the Supplementary Appendix). A similar pattern was seen for myocardial infarction (Table S7 in the Supplementary Appendix). There was no consistent pattern for stroke or heart failure.

The overall rates of major cardiovascular events showed a pattern similar to that for mortality, with rates of 3.99, 5.38, and 6.43 events per 1000 person-years in high-, middle-, and low-income countries, respectively $(\mathrm{P}<0.001)$ (Fig. 2, and Table S7 in the Supplementary Appendix). By contrast, nonmajor cardiovascular events (cardiovascular events, other than major cardiovascular events, that required hospitalization) showed the opposite pattern, with event rates of 3.69, 1.72, and 1.10 per 1000 person-years in high-, middle-, and low-income countries, respectively $(\mathrm{P}<0.001)$. The most common nonmajor cardiovascular events are shown in Table S8 in the Supplementary Appendix.

\section{ASSOCIATION BETWEEN INTERHEART RISK SCORE AND CARDIOVASCULAR EVENTS}

The INTERHEART Risk Score predicted cardiovascular events (data not shown); however, within each stratum of the INTERHEART Risk Score, the rates of major cardiovascular events and of 


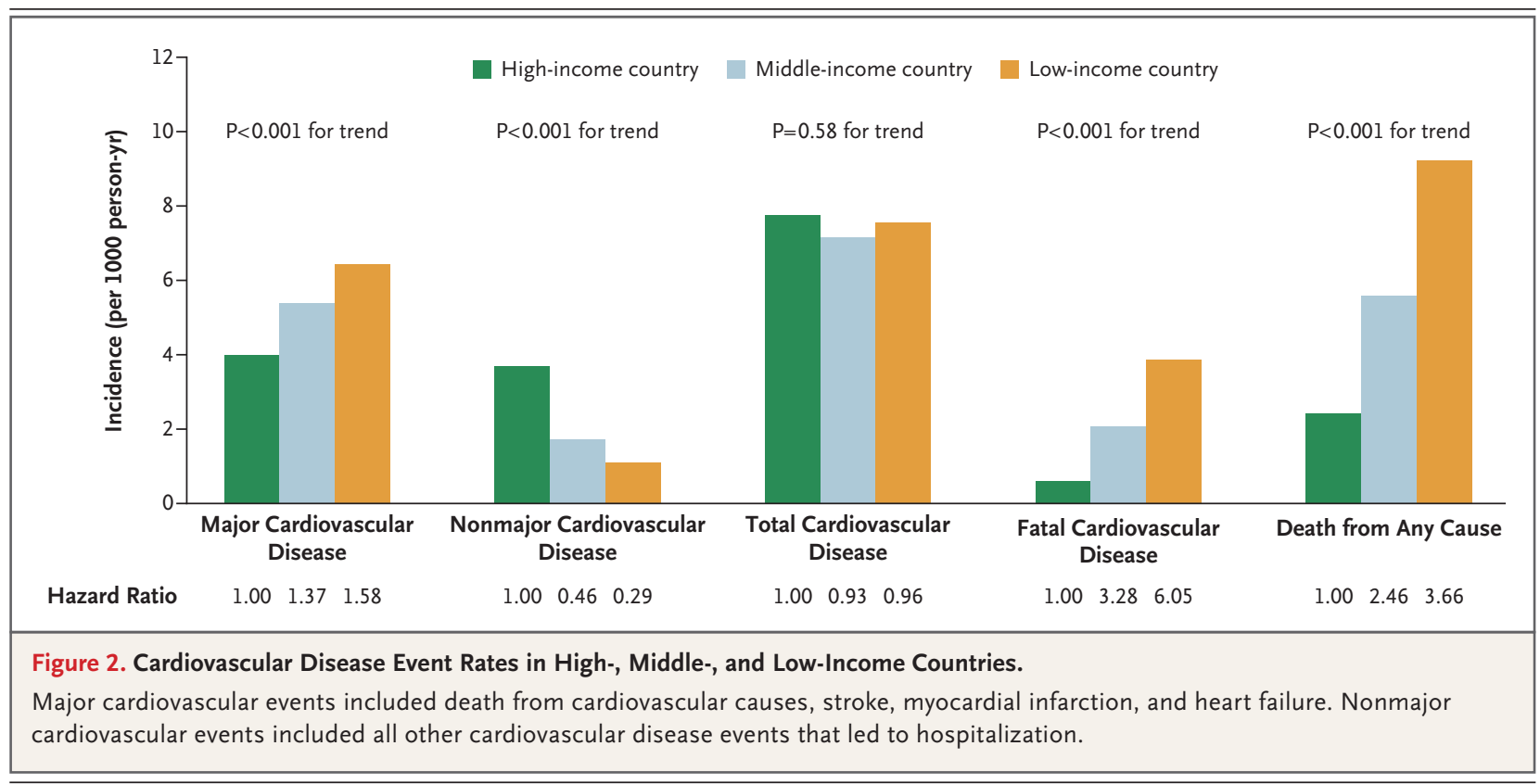

death were lowest in high-income countries, intermediate in middle-income countries, and highest in low-income countries (data not shown). After adjustment for variations in the INTERHEART Risk Score among the three income strata, the rates of major cardiovascular events were 3.64, 5.23, and 7.39 per 1000 person-years in high-, middle-, and low-income countries, respectively $(\mathrm{P}<0.001)$, and the rates of death were 2.19, 5.50, and 9.84 per 1000 person-years, respectively $(\mathrm{P}<0.001)$.

\section{CASE FATALITY RATES ACCORDING TO COUNTRY INCOME STRATUM}

The proportions of participants with major cardiovascular disease who died (the case fatality rates) were higher in low- and middle-income countries than in high-income countries $(6.50 \%$ in high-income countries, $15.86 \%$ in middle-income countries, and $17.28 \%$ in low-income countries; $\mathrm{P}=0.01$ ) (Fig. 3, and Table S9 in the Supplementary Appendix). This relationship was consistent among patients with myocardial infarction, those with stroke, and those with heart failure.

CARDIOVASCULAR EVENTS AND CASE FATALITY RATES ACCORDING TO URBAN VERSUS RURAL LOCATION

The rate of major cardiovascular events was lower in urban communities than in rural communities (4.83 events vs. 6.25 events per 1000 person-years, $\mathrm{P}<0.001$ ) (Fig. 4A), as were the rates of fatal cardiovascular events (1.71 events vs. 3.09 events per 1000 person-years, $\mathrm{P}<0.001)$ and of deaths from any cause (4.48 deaths vs. 8.01 deaths per 1000 person-years, $\mathrm{P}<0.001$ ) (Table $\mathrm{S} 10$ in the Supplementary Appendix). The rate of nonmajor cardiovascular events showed the opposite trend (2.12 events per 1000 person-years in urban communities vs. 1.50 events per 1000 person-years in rural communities, $\mathrm{P}<0.001)$. In both high-income and low-income countries, case fatality rates were similar in rural and urban areas, but in middleincome countries, the rates were higher in rural areas than in urban areas (Fig. 4B, and Table S11 in the Supplementary Appendix).

\section{DISCUSSION}

Our study has three major findings. First, the incidence of major cardiovascular events was highest in low-income countries, despite the fact that these countries had the lowest risk-factor burden. In contrast, the incidence of nonmajor cardiovascular events was highest in high-income countries. Second, case fatality rates after major cardiovascular events and the rates of death from any cause were highest in low-income countries, intermediate in middle-income countries, and lowest in high-income countries. Third, the rates of major cardiovascular events and the rates of death from any cause were higher in rural communities than in urban communities in middle-income countries and low-income countries, whereas the risk-factor burden was higher in the urban com- 


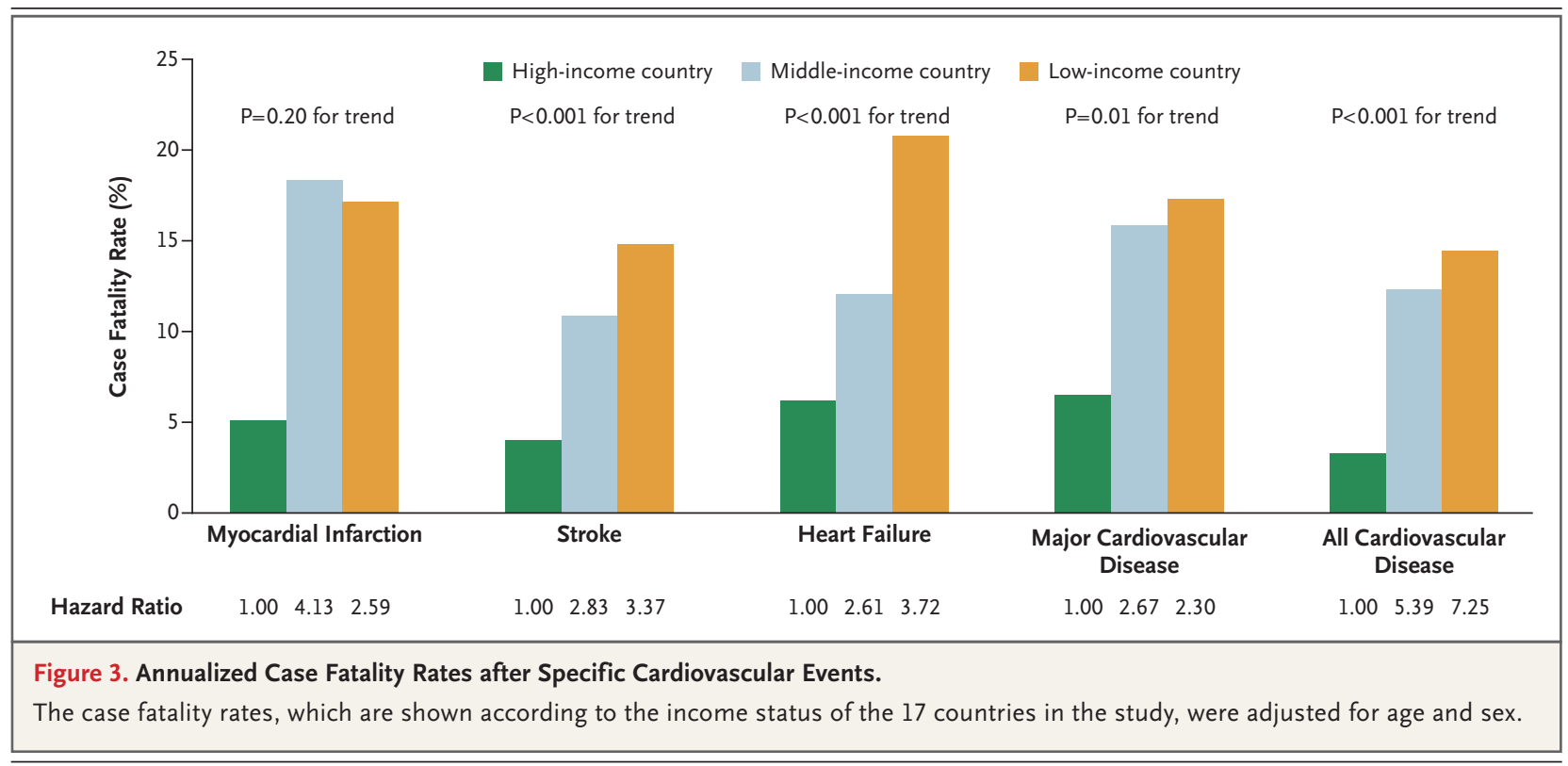

munities than in the rural communities in those countries.

The version of the INTERHEART Risk Score that is calculated without the use of laboratory testing was developed with data from the INTERHEART study for use in resource-limited settings. It has been validated in a cohort selected from 21 countries $^{15}$ and predicts cardiovascular disease and mortality in the PURE study (data not shown). However, within each stratum of risk score, the rates of major cardiovascular events and of death were lowest in high-income countries, intermediate in middle-income countries, and highest in low-income countries, whereas for the rates of nonmajor cardiovascular events, the reverse trend was seen. These findings indicate that influences other than risk factors are important in determining outcomes at the country level. Possible contributing factors could include access to and affordability of health services and medications, thresholds for diagnoses and treatments, and educational level of the population. This interpretation is supported by parallel observations in the PURE study of the greater use of preventive drugs and coronary revascularization, better control of hypertension, and lower current smoking rates (all markers of better health care systems) in high-income countries, ${ }^{9,10}$ which may mitigate higher riskfactor levels in those countries.
The similarity in the rates of major cardiovascular disease and deaths among urban and rural participants in high-income countries may reflect the structure of the health systems in the three high-income countries we studied, with universal coverage ensuring good access in both urban and rural areas. By contrast, in most middleand low-income countries, there may be greater differences between urban and rural communities in the educational level of the population as well as in the access to and quality and affordability of health care, which may contribute to higher rates of death from cardiovascular causes in rural areas, despite a lower risk-factor burden. These data therefore also suggest that health care systems are likely to have a large impact on cardiovascular disease outcomes.

Most previous epidemiologic studies of cardiovascular risk, including the landmark Multinational Monitoring of Trends and Determinants in Cardiovascular Disease (MONICA) study, ${ }^{16}$ were conducted largely in wealthier countries (mainly Europe; see Fig. S2 in the Supplementary Appendix), although the MONICA study and some other studies have included a few countries, such as China, that are at lower economic levels. ${ }^{17}$ The challenges of conducting a study that includes countries across a wide range of socioeconomic circumstances are considerable. However, such studies may provide important information that 
cannot be obtained from analyses of single countries, especially if those analyses are confined to wealthy regions.

Our study has several limitations. First, it was not considered to be feasible to use strict proportionate sampling in each country; therefore, caution is needed in interpreting our data as being representative of each country. However, the countries included are not atypical with respect to the range of economic levels (Fig. S1 in the Supplementary Appendix); in addition, the risk-factor levels in each country in the PURE study parallel those in previous studies, the populations that were included in our study were similar to those that were eligible for inclusion, and the rates of death in each household in the 2 years before inclusion in the study correlated closely with national rates of death and with those observed during follow-up (Fig. S3 and S4 in the Supplementary Appendix).

Second, we were able to follow only $92.8 \%$ of our participants $(97.5 \%$ of those in high-income countries, $94.4 \%$ of those in middle-income countries, and $85.9 \%$ of those in low-income countries). Follow-up rates were high in the rural areas of all countries and in the urban areas of high- and middle-income countries but were lower in the urban areas of low-income countries $(76.8 \%)$. The rates in urban areas of low-income countries may have been lower because some housing areas were demolished or communities dispersed owing to social and economic unrest. Given the small proportion of participants for whom follow-up data were not available, adjustment for the differences observed in INTERHEART risk scores and educational level between participants with and those without follow-up data did not materially alter our conclusions.

Third, the detection of cardiovascular events (though not of death) may have been hampered by differences in access to hospital and diagnostic facilities between lower-income countries and higher-income countries. However, if event detection were limited to a substantial extent because of reduced access in lower-income countries, it would imply that the rates of cardiovascular events are actually even higher in those countries.

In conclusion, our large study of cardiac risk factors and cardiovascular events among adults in urban and rural communities in low-, middle-,

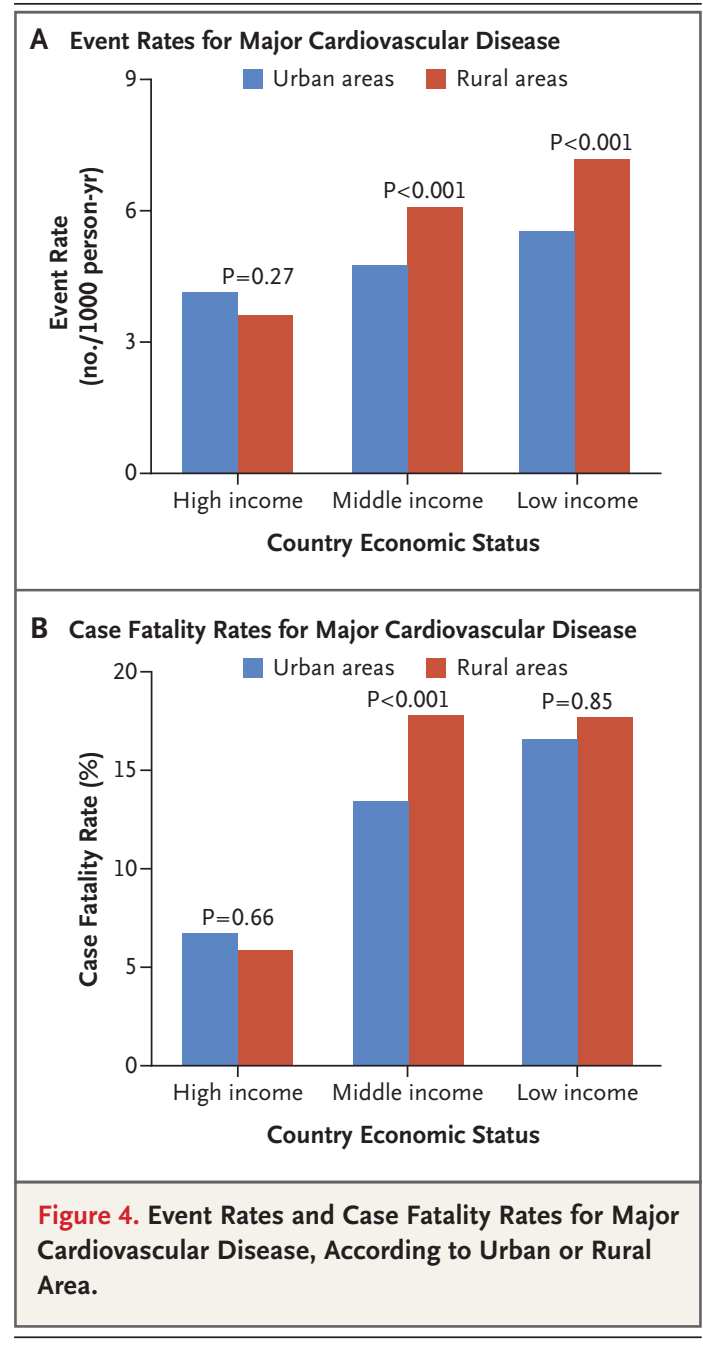

and high-income countries on five continents showed that the incidence of major cardiovascular disease was highest in low-income countries, despite the fact that these countries had the lowest risk-factor burden. Case fatality rates were also highest in the low-income countries. Rural communities had a lower risk-factor burden than urban communities but higher cardiovascularevent rates and case fatality rates.

Supported by the Population Health Research Institute; the Canadian Institutes of Health Research; the Heart and Stroke Foundation of Ontario; unrestricted grants from several pharmaceutical companies (with major contributions from AstraZeneca [Canada], Sanofi-Aventis [France and Canada], Boehringer Ingelheim [Germany and Canada], Servier, and GlaxoSmithKline and additional contributions from Novartis and King Pharma), and various national or local organizations in participating countries, as follows: Fundacion Estudios Clínicos Latinoamérica (Argentina); Independent University, Bangladesh, and Mitra and Associates (Bangladesh); Unilever Health Institute (Brazil); Public Health Agency of Canada and Champlain Cardiovascular Disease 
Prevention Network (Canada); Universidad de La Frontera (Chile); National Center for Cardiovascular Diseases (China); a grant (6566-04-18062) from Colciencias (Colombia); Indian Council of Medical Research (India); grants from the Ministry of Science, Technology and Innovation of Malaysia (100-IRDC/ BIOTEK 16/6/21 [13/2007] and 07-05-IFN-BPH 010), the Ministry of Higher Education of Malaysia (600-RMI/LRGS/5/3 [2/2011]), Universiti Teknologi MARA, Universiti Kebangsaan Malaysia (UKM-Hejim-Komuniti-15-2010) (Malaysia); the Polish Ministry of Science and Higher Education (Nr 290/W-PURE/2008/0) and Wroclaw Medical University (Poland); the North-West University, South Africa and the Netherlands Program for Alternative Development, National Research Foundation, Medical Research
Council of South Africa, the South African Sugar Association, Faculty of Community and Health Sciences (South Africa); AFA Insurance, Swedish Council for Working Life and Social Research, Swedish Research Council for Environment, Agricultural Sciences and Spatial Planning, Swedish Heart and Lung Foundation, Swedish Research Council, a grant from the Swedish State (Läkarutbildnings Avtalet), a grant from the Västra Götaland Region (FOUU) (Sweden); the Metabolic Syndrome Society, AstraZeneca, and Sanofi-Aventis (Turkey); and Sheikh Hamdan Bin Rashid Al Maktoum Award for Medical Sciences and Dubai Health Authority, Dubai (United Arab Emirates).

Disclosure forms provided by the authors are available with the full text of this article at NEJM.org.

APPENDIX

The authors' full names and academic degrees are as follows: Salim Yusuf, M.B., B.S., D.Phil., Sumathy Rangarajan, M.Sc., Koon Teo, M.B., Ph.D., Shofiqul Islam, M.Sc., Wei Li, Ph.D., Lisheng Liu, M.D., Jian Bo, B.Sc., Qinglin Lou, M.Sc., Fanghong Lu, B. Sc., Tianlu Liu, B.Sc., Liu Yu, B.Sc., Shiying Zhang, B.Sc., Prem Mony, M.D., Sumathi Swaminathan, Ph.D., Viswanathan Mohan, M.D., Rajeev Gupta, M.D., Ph.D., Rajesh Kumar, M.D., Krishnapillai Vijayakumar, M.D., Scott Lear, Ph.D., Sonia Anand, M.D., Ph.D., Andreas Wielgosz, M.D., Ph.D., Rafael Diaz, M.D., Alvaro Avezum, M.D., Ph.D., Patricio Lopez-Jaramillo, M.D., Ph.D., Fernando Lanas, M.D., Khalid Yusoff, M.B., B.S., Noorhassim Ismail, M.D., Ph.D., Romaina Iqbal, Ph.D., Omar Rahman, M.D., M.P.H., D.Sc., Annika Rosengren, M.D., Afzalhussein Yusufali, M.D., Roya Kelishadi, M.D., Annamarie Kruger, Ph.D., Thandi Puoane, M.P.H., Ph.D., Andrzej Szuba, M.D., Ph.D., Jephat Chifamba, M.Phil., Aytekin Oguz, M.D., Matthew McQueen, M.B., Ch.B., Martin McKee, D.Sc., and Gilles Dagenais, M.D., for the PURE Investigators

The authors' affiliations are as follows: the Population Health Research Institute, McMaster University and Hamilton Health Sciences, Hamilton, ON (S.Y., S.R., K.T., S.I., S.A., M. McQueen), Department of Biomedical Physiology and Kinesiology, Simon Fraser University, Burnaby, BC (S.L.), the Department of Medicine, University of Ottawa, Ottawa, ON (A.W.), and Laval University Heart and Lungs Institute, Quebec City, QC (G.D.) — all in Canada; the National Center for Cardiovascular Diseases, Cardiovascular Institute and Fuwai Hospital, Chinese Academy of Medical Sciences, Beijing (W.L., L.L., J.B.), Jiangsu Province Institute of Geriatrics, Jiangsu Province, Nanjing City (Q.L.), Shandong Province Academy of Medical Science, Shandong Province, Jinan City (F. Lu), Xi'an Electronic Technology University Hospital, Shanxi Province, Xi'an City (T.L.), Shenyang City 242 Hospital, Liaoning Province, Shenyang City, Huanggu District (L.Y.), Bayannaoer Center for Disease Control and Prevention, Inner Mongolia, Bayannaoer City, Linhe District, Jiefangxi (S.Z.) — all in China; the Division of Epidemiology and Population Health, St. John's Research Institute, Bangalore (P.M., S.S.), Madras Diabetes Research Foundation, Chennai (V.M.), Fortis Escorts Hospitals, JLN Marg, Jaipur (R.G.), Postgraduate Institute of Medical Education and Research School of Public Health, Chandigarh (R. Kumar), and Health Action by People, Trivandrum, Kerala (K.V.) — all in India; Estudios Clinicos Latinoamerica ECLA, Rosario, Santa Fe, Argentina (R.D.); Dante Pazzanese Institute of Cardiology, São Paulo (A.A.); Fundacion Oftalmologica de Santander (FOSCAL), Medical School, Universidad de Santander, Floridablanca-Santander, Colombia (P.L.-J.); Universidad de La Frontera, Temuco, Chile (F. Lanas); Faculty of Medicine, Universiti Teknologi MARA, Sungai Buloh, Selangor, and UCSI University Kuala Lumpur, Kuala Lumpur (K.Y.), and the Department of Community Health, University Kebangsaan Malaysia Medical Centre, Kuala Lumpur (N.I.) - all in Malaysia; the Departments of Community Health Sciences and Medicine, Aga Khan University, Stadium Road, Karachi, Pakistan (R.I.); Independent University, Dhaka, Bangladesh (O.R.); Sahlgrenska Academy, University of Gothenburg, Gothenburg, Sweden (A.R.); Hatta Hospital, Dubai Health Authority, Dubai, United Arab Emirates (A.Y.); Isfahan Cardiovascular Research Center, Isfahan Cardiovascular Research Institute, Isfahan University of Medical Sciences, Isfahan, Iran (R. Kelishadi); Faculty of Health Science North-West University, Potchefstroom Campus, Potchefstroom (A.K.), and the School of Public Health, University of the Western Cape, Bellville (T.P.) — both in South Africa; Wroclaw Medical University, Department of Internal Medicine, Borowska, Wroclaw, Poland (A.S.); University of Zimbabwe, College of Health Sciences, Physiology Department, Harare (J.C.); Istanbul Medeniyet University, Faculty of Medicine, Department of Internal Medicine Istanbul, Turkey (A.O.); and the London School of Hygiene and Tropical Medicine, London (M. McKee).

\section{REFERENCES}

1. Murray CJL, Vos T, Lozano R, et al. Disability-adjusted life years (DALYs) for 291 diseases and injuries in 21 regions, 1990-2010: a systematic analysis for the Global Burden of Disease Study 2010. Lancet 2012;380:2197-223.

2. Walker AR, Walker BF, Segal I. Some puzzling situations in the onset, occurrence and future of coronary heart disease in developed and developing populations, particularly such in sub-Saharan Africa. J R Soc Promot Health 2004;124: 40-6.

3. Marmot M. Coronary heart disease: rise and fall of a modern epidemic. In: Marmot M, Elliot P, eds. Coronary heart disease epidemiology: from aetiology to public health. Oxford, United Kingdom: Oxford University Press, 1992:3-19.

4. O'Flaherty M, Buchan I, Capewell S. Contributions of treatment and lifestyle to declining CVD mortality: why have CVD mortality rates declined so much since the 1960s? Heart 2013;99:159-62.

5. Stringhini S, Viswanathan B, Gédéon J, Paccaud F, Bovet P. The social transition of risk factors for cardiovascular disease in the African region: evidence from three cross-sectional surveys in the Seychelles. Int J Cardiol 2013;168:1201-6.

6. Krishnamurthi RV, Feigin VL, Forouzanfar MH, et al. Global and regional bur- den of first-ever ischaemic and haemorrhagic stroke during 1990-2010: findings from the Global Burden of Disease Study 2010. Lancet Glob Health 2013;1(5):e259e281.

7. Teo K, Chow CK, Vaz M, Rangarajan S, Yusuf S. The Prospective Urban Rural Epidemiology (PURE) study: examining the impact of societal influences on chronic noncommunicable diseases in low-, middle-, and high-income countries. Am Heart J 2009;158(1):1.e1-7.e1.

8. Corsi DJ, Subramanian SV, Chow CK, et al. Prospective Urban Rural Epidemiology (PURE) study: baseline characteristics of the household sample and com- 
parative analyses with national data in 17 countries. Am Heart J 2013;166:636-46

9. Yusuf S, Islam S, Chow CK, et al. Use of secondary prevention drugs for cardiovascular disease in the community in high-income, middle-income, and lowincome countries (the PURE Study): a prospective epidemiological survey. Lancet 2011:378:1231-43.

10. Chow CK, Teo KK, Rangarajan S, et al. Prevalence, awareness, treatment, and control of hypertension in rural and urban communities in high-, middle-, and low-income countries. JAMA 2013;310: 959-68.

11. Yusuf S, Hawken S, Ounpuu S, et al. Effect of potentially modifiable risk factors associated with myocardial infarc- tion in 52 countries (the INTERHEART study): case-control study. Lancet 2004; 364:937-52.

12. O’Donnell MJ, Xavier D, Liu L, et al. Risk factors for ischaemic and intracerebral haemorrhagic stroke in 22 countries (the INTERSTROKE study): a case-control study. Lancet 2010;376:112-23.

13. Gajalakshmi V, Peto R. Verbal autopsy of 80,000 adult deaths in Tamilnadu, South India. BMC Public Health 2004;4: 47.

14. Jha P, Gajalakshmi V, Gupta PC, et al. Prospective study of one million deaths in India: rationale, design, and validation results. PLoS Med 2006;3(2):e18.

15. McGorrian C, Yusuf S, Islam S, et al. Estimating modifiable coronary heart disease risk in multiple regions of the world: the INTERHEART Modifiable Risk Score. Eur Heart J 2011;32:581-9.

16. Tunstall-Pedoe H, Vanuzzo D, Hobbs $\mathrm{M}$, et al. Estimation of contribution of changes in coronary care to improving survival, event rates, and coronary heart disease mortality across the WHO MONICA Project populations. Lancet 2000;355: 688-700.

17. Wu Z, Yao C, Zhao D, et al. Cardiovascular disease risk factor levels and their relations to CVD rates in China - results of Sino-MONICA project. Eur J Cardiovasc Prev Rehabil 2004;11:275-83.

Copyright @ 2014 Massachusetts Medical Society. 